\title{
TASAS DE GENERACIÓN Y CARACTERIZACIÓN DE RESIDUOS SÓLIDOS ORDINARIOS EN CUATRO MUNICIPIOS DEL ÁREA METROPOLITANA COSTA RICA
}

\author{
GENERATION RATES AND CHARACTERIZATION OF \\ ORDINARY SOLID WASTE IN FOUR MUNICIPALITIES \\ OF THE METROPOLITAN AREA COSTA RICA
}

\author{
Jorge Herrera-Murillo \\ José Félix Rojas-Marin ${ }^{2}$ \\ Deivis Anchía-Leitón ${ }^{3}$ \\ Universidad Nacional, Heredia, Costa Rica
}

\section{RESUMEN}

Se determinó la tasa de generación y caracterización de residuos sólidos ordinarios, en el sector residencial y comercial de 04 municipios ubicados geográficamente dentro del Gran Área Metropolitana de Costa Rica. Para ello, en el período 2014-2015, se realizaron muestreos en un número representativo de viviendas y comercios durante 07 días consecutivos. La tasa de generación promedio ponderada de residuos para el área de estudio resultó ser de $0,59 \mathrm{~kg} / \mathrm{hab}$-día. Los residuos sólidos generados presentan como componentes mayoritarios: orgánicos $55,9 \%$, seguidos de materiales

1 Académico y coordinador del Laboratorio de Análisis Ambiental, Universidad Nacional, Heredia, Costa Rica. Correo electrónico: jorge.herrera.murillo@una.cr

2 Académico e investigador del Laboratorio de Análisis Ambiental, Universidad Nacional, Heredia, Costa Rica. Correo electrónico: jose.rojas.marin@una.cr

3 Académico e investigador del Laboratorio de Análisis Ambiental, Universidad Nacional, Heredia, Costa Rica. Correo electrónico: deivis.anchia.leiton@una.cr

Fecha de recepción: 28 de junio de 2016

Fecha de aceptación: 18 de julio de 2016 
con alto potencial para ser reciclados o utilizados como combustibles (plásticos 10,2\%, papel y cartón 10,4\%). El contenido de humedad promedio en los residuos fue de 58,7\% en masa (mínimo $54 \%$ en Alajuela y máximo $63 \%$ en Barva), de la cual hasta un $67 \%$ puede ser atribuible a residuos orgánicos biodegradables. El peso volumétrico promedio de los residuos resultó de $155 \mathrm{~kg} /$ $\mathrm{m}^{3}$ (mínimo de 141 en Belén y máximo de 163 en San José). Se evidenció que existen diferencias significativas en la composición de residuos sólidos para los sectores comerciales y residenciales, principalmente en la fracción orgánica, papel, cartón y plástico.

Palabras clave: residuos sólidos ordinarios, tasas de generación, gran área metropolitana de Costa Rica

\begin{abstract}
Generation rates and the characterization of ordinary solid waste were determined in the residential and commercial sectors of four municipalities in the Metropolitan Area of Costa Rica. For this, a sampling was performed on a representative number of homes and businesses in the 2014-2015 period during seven days. The waste generation weighted average rate in the work area turned out to be $0.59 \mathrm{~kg}$ / inhabitants.-day. Solid waste generated presented as major components: organic $55.9 \%$, followed by materials with high potential to be recycled or used as fuel $(10.2 \%$ plastic, paper and cardboard $10.4 \%$ ). The average moisture content in the waste was 58.7 mass\% (minimum $54 \%$ in Alajuela and maximum $63 \%$ in Barva), of which up to $67 \%$ may be attributable to biodegradable organic waste. The average waste volumetric weight was $155 \mathrm{~kg} / \mathrm{m}^{3}$ (minimum of 141 in Belen and maximum of 163 in San Jose). Significant differences appear in the composition of commercial and residential waste, mainly regarding organic waste, paper, cardboard, and plastic.
\end{abstract}

Keywords: Urban solid waste, generation rates, metropolitan area

\title{
Introducción
}

Hasta hace algunos años, el manejo de los residuos sólidos en Costa Rica se consideraba un problema menor circunscrito a escala local, que involucraba la participación de los municipios y algunas pocas entidades públicas y privadas. Sin embargo, el acelerado crecimiento demográfico, la concentración e incremento poblacional en áreas urbanas y un modelo económico que potencia los hábitos de consumo en los ciudadanos han generado que el manejo de los residuos sólidos, desde la recolección hasta la disposición final, se haya constituido en uno de los problemas ambientales más trascendentales a nivel nacional. Lo anterior ocasiona implicaciones importantes en la planificación urbana de los principales centros poblados del país, de la misma forma que ocurre en la mayoría de las naciones en vías de desarrollo (Hoornweg y Bhada-Tata, 2012).

En la gestión integral de residuos intervienen distintos actores que interactúan ocupando espacios geográficos y administrativos en diferentes niveles de participación, posibilitando una escala de análisis variada. Estos puntos de interpretación dan cuenta de la complejidad del sistema 
completo de los residuos y en el cual se introduce la geografía como ciencia y disciplina, con la posibilidad de contribuir a su análisis desde una dimensión temporal y espacial.

Costa Rica ha venido incorporando dentro de la legislación una serie de regulaciones en pro del desarrollo de un sistema de gestión integral de residuos sólidos. Uno de los primeros esfuerzos fue la aprobación del Plan Nacional de Residuos Sólidos en el año 1991; sin embargo, el mismo no dio los resultados esperados (Programa CYMA, 2007). Aproximadamente, dos décadas después, se crea la Política Nacional para la Gestión Integral de Residuos Sólidos 2010-2021, la cual originó la promulgación de la Ley No. 8839, donde se obliga específicamente a los gobiernos locales a responsabilizarse por el manejo de residuos producidos dentro del territorio cantonal. El articulo 8 indica textualmente: "Las municipalidades serán responsables de la gestión integral de los residuos generados en su cantón; para ello deberán establecer y aplicar el plan municipal para la gestión integral de residuos en concordancia con la política y el plan nacional" (La Gaceta, 2016).

Los residuos son todos aquellos materiales que se encuentran en estado sólido, semisólido, líquido o gas contenidos en recipientes que resultan de diferentes actividades de extracción, transformación, producción, consumo y tratamiento, cuya condición actual no permite su utilización nuevamente en el proceso que lo originó, y que puede ser susceptible a ser valorizado o requiere tratamiento o disposición final para evitar un impacto negativo en el ambiente (SEMARNAT, 2006). Dentro de esta categoría, los residuos sólidos ordinarios son definidos como: "los generados en las casas de habitación, los cuales resultan de la eliminación de los materiales que se utilizan en las actividades domésticas, de los productos que consumen y de sus envases, embalajes o empaques; de igual forma se incluyen aquellos que provienen de cualquier otra actividad realizada en establecimientos o en la vía pública que posean características domiciliarias" (Castillo, 2013, p.14).

La problemática ambiental generada por el incremento de los residuos sólidos en Costa Rica se debe, en parte, a la ausencia de un modelo de desarrollo urbano que promueva la gestión integral de los residuos sólidos (GIRS), ya que esta implica un conjunto interrelacionado de acciones normativas, operativas, financieras, administrativas, sociales, educativas, 
de monitoreo y supervisión para el manejo de los residuos, desde su generación hasta la disposición final, a fin de lograr beneficios ambientales, la optimización económica de su manejo, respondiendo a las necesidades y circunstancias de cada región (Programa CYMA, 2007).

El análisis y localización espacial de la generación de residuos y su variación a través del tiempo, constituye un elemento importante para la GIRS y el ordenamiento territorial del país, es la base a partir de la cual se logra el diseño de un sistema de manejo de residuos que atienda las necesidades actuales de los diferentes centros urbanos. La generación de residuos sólidos en distintos espacios geográficos, está relacionada con parámetros como nivel socioeconómico, educación, cantidad de habitantes, número de establecimientos comerciales, disposiciones ambientales y el nivel cultural de la población, entre otros (Lund y Muenster, 2010).

De igual forma, como parte del diseño del sistema de GIRS debe construirse una base de datos descriptiva de los residuos sólidos por tratar, sobre todo en cuanto a su segregación en materiales y cantidades producidas. Las propiedades físicas, como la densidad o contenido de humedad pueden ser de utilidad para indicar las condiciones de transporte, requerimientos de su procesamiento, características de combustión y una predicción aproximada en cuanto al tiempo de vida de un relleno sanitario (López, 1999). Entre las principales propiedades físicas y químicas de los residuos sólidos por considerar para el desarrollo de un sistema de GIRS se encuentran:

- $\quad$ Peso volumétrico: se refiere al peso de los residuos sólidos contenidos en una unidad de volumen. En el caso de los países de América Latina y el Caribe, el peso específico o densidad alcanza valores de 125 a $250 \mathrm{~kg} / \mathrm{m}^{3}$ (Jaramillo, 2002).

- Humedad: corresponde a la pérdida de agua que se genera cuando se calientan los residuos a $105^{\circ} \mathrm{C}$ durante una hora. Puede expresarse como el porcentaje del peso del material húmedo. En el caso de los países de América Latina y el Caribe, los residuos tienen un mayor contenido de materia orgánica y una humedad que varía de 35 a $55 \%$ (Jaramillo 2002). Conocer el valor del contenido de humedad, es útil para estimar la cantidad de calor que se recuperara a partir de ellos, ya que si el contenido en humedad es alto mucha energía 
será consumida en eliminar el agua. Además la humedad debe ser tomada en cuenta para diseñar los requerimientos necesarios para su transporte (Vértice, 2008).

- Cenizas: es el residuo sólido resultante de la combustión de productos carbonáceos. Es un factor que se debe considerar para el diseño de un tratamiento térmico, ya que en caso de que los residuos tengan un alto contenido de cenizas, el tren de tratamiento de emisiones a la atmosfera tendría que considerarlo en su diseño (Tchobanoglous, Theisen y Vigil, 1994).

El presente trabajo muestra los resultados de los estudios de generación, segregación y composición de los residuos sólidos ordinarios generados en 04 municipios del territorio nacional. La información obtenida se analiza desde la perspectiva de las variables de influencia tomando en cuenta la variabilidad temporal y espacial de los datos. El nivel de cobertura y la alta representatividad de los datos permitirán orientar la toma de decisiones en materia de GIRS y ordenamiento territorial, tanto en los municipios participantes como en los restantes pertenecientes al Gran Área Metropolitana de Costa Rica (GAM).

\section{Metodología}

\section{a. Muestreo}

Se seleccionaron 04 municipios del GAM, a saber: San José, Alajuela, Belén y Barva (Figura 1). Dichos cantones fueron elegidos utilizando criterios de densidad poblacional, patrones de uso del suelo y grado de industrialización. En cada uno de estos se realizaron muestreos tanto en el sector comercial como residencial. Para este último, se tomó como punto de partida los datos del número de viviendas por distrito reportados por el Instituto Nacional de Estadísticas y Censos. El sector residencial fue dividido en tres estratos: alto, medio y bajo aplicando los criterios del Departamento de Catastro de cada una las municipalidades seleccionadas y la Dirección General de Tributación Directa del Ministerio de Hacienda, para proceder posteriormente, a la ubicación de los estratos en los mapas del cantón donde se registraron todas y cada una de las unidades muestrales. 
Figura 1. Ubicación geográfica, cantones muestreados, 2014-2015

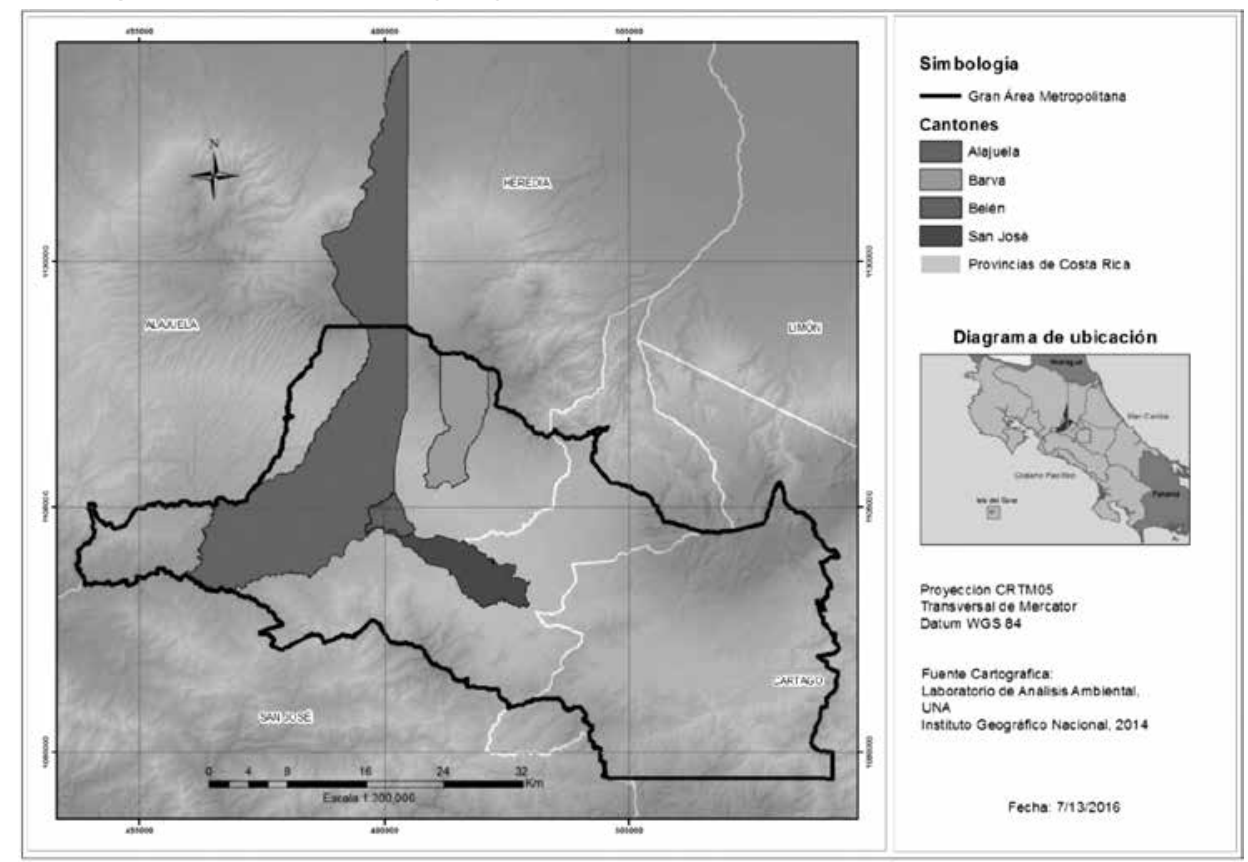

Fuente: Elaboración propia.

Para la estimación de la cantidad de viviendas por muestrear en cada uno de los cantones, se utilizó la siguiente fórmula estadística, según la guía del CYMA (2012):

$$
n_{v i v}=\left(\frac{Z^{2} \times N_{v i v} \times \delta^{2}}{\left(N_{v i v}-1\right) \times E^{2}+\left(Z^{2} \times \delta^{2}\right)}\right) \times 1,25
$$

Donde:

$n_{v i v}=$ número de viviendas que incluirá la muestra

$N_{\text {viv }}=$ número de viviendas en el área de estudio

$Z=$ coeficiente de confianza al $95 \%(1,96)$

$\delta=$ desviación estándar $(0,3 \mathrm{~kg} / \mathrm{hab} /$ día $)$

$E=$ error permisible $(0,05 \mathrm{~kg} / \mathrm{hab} / \mathrm{dia})$ 
Es importante señalar que el cálculo de la muestra se realizó tomando en cuenta un $25 \%$ más de lo necesario, esto con el fin de subsanar las pérdidas de muestra. Además, después de aplicar esta fórmula, el valor domiciliar se dividió con respecto a la distribución de los estratos sociales para el cantón en específico. Los valores resultantes se muestran en el cuadro 1.

Por otro lado, para la selección del tamaño de muestra en el sector comercial, se utilizó como punto de partida el listado de patentes de cada uno de los municipios, así como la ecuación (2):

$$
n_{\text {com }}=\left(\frac{Z^{2} \times N_{\text {com }} \times \delta^{2}}{\left(N_{c o m}-1\right) \times E^{2}+\left(Z^{2} \times \delta^{2}\right)}\right) \times 1,25
$$

Donde:

$$
\begin{aligned}
& n_{\text {com }}=\text { número de comercios que incluirá la muestra } \\
& N_{\text {com }}=\text { número de comercios en el área de estudio } \\
& Z=\text { coeficiente de confianza al } 95 \%(1,96) \\
& \delta=\text { desviación estándar }(0,5 \mathrm{~kg} / \mathrm{hab} / \text { día }) \\
& E=\text { error permisible }(0,15 \mathrm{~kg} / \mathrm{hab} / \mathrm{dia})
\end{aligned}
$$

Para el sector comercial, se realizó una subdivisión por tipo de actividad comercial, tomando en cuenta el código de la Clasificación Industrial Internacional Uniforme (CIIU) según detalle mostrado en el cuadro 2. El detalle de la cantidad de sitios de muestreo incluidos por cantón en el sector comercial se muestra en el cuadro 3.

Cuadro 1. Distribución de las viviendas muestreadas por estrato en cada uno de los cantones comprendidos en el estudio.

\begin{tabular}{|l|c|c|c|}
\hline \multicolumn{1}{|c|}{ Municipio } & $\begin{array}{c}\text { Viviendas de estrato } \\
\text { bajo }\end{array}$ & $\begin{array}{c}\text { Viviendas de estrato } \\
\text { medio }\end{array}$ & $\begin{array}{c}\text { Viviendas de estrato } \\
\text { alto }\end{array}$ \\
\hline San José & 1010 & 2557 & 528 \\
\hline Alajuela & 112 & 38 & 23 \\
\hline Belén & 47 & 102 & 62 \\
\hline Barva & 109 & 39 & 23 \\
\hline Total & $\mathbf{1 2 7 8}$ & $\mathbf{2 7 3 6}$ & $\mathbf{6 3 6}$ \\
\hline
\end{tabular}

Fuente: Elaboración propia. 
Para la identificación de los sitios de muestreo, tanto para el sector domiciliar como comercial, se estableció un código según la naturaleza del punto; este constaba de 3 secciones; la primera de ellas se refería al distrito correspondiente; la segunda para el sector domiciliar, la clase social y para el sector comercial, el tipo de comercio; y la última sección del mismo código se refiere al consecutivo de muestra.

\section{Cuadro 2. Codificación utilizada para la selección de la muestra en el sector comercial}

\begin{tabular}{|c|c|c|}
\hline Código & Clasificación & Detalle \\
\hline 04 & Otros servicios: Financieros & Agencia bancaria \\
\hline 05 & Otros servicios: Religiosos & Iglesias católicas, no católicas, congregaciones \\
\hline 06 & Otros servicios: Transporte & Parqueos \\
\hline 07 & Industria o producción & Fábricas \\
\hline 08 & Almacén o depósito & Bodegas de almacenamiento y distribución \\
\hline 09 & Asistencia social & Albergues, hogares para niños, ancianos o indigentes \\
\hline 10 & Información y Comunicación & Estación de radio, producción de programas de radio \\
\hline 11 & Abarrotes & $\begin{array}{l}\text { Pulpería, minisúper, supermercado, carnicería, pescadería, } \\
\text { verdulería }\end{array}$ \\
\hline 12 & Comercio general & $\begin{array}{l}\text { Boutique, librería, floristería, joyería, celulares, pañalera, } \\
\text { bazar, licorera, casa de empeño, mueblería, venta de } \\
\text { materiales de construcción, venta de loterías, chances }\end{array}$ \\
\hline 13 & Comidas y bebidas & $\begin{array}{l}\text { Restaurantes, sodas, comidas rápidas, bar, panaderías, } \\
\text { pizzería, heladería }\end{array}$ \\
\hline 14 & Entretenimiento & $\begin{array}{l}\text { Internet café, gimnasio, billar, boliche, video, cine, salón } \\
\text { comunal, biblioteca, sala de juegos }\end{array}$ \\
\hline 15 & Hospedaje & Albergue, hotel, motel \\
\hline 16 & Asociaciones & $\begin{array}{l}\text { Oficinas de cámara o asociación, asociación empresarial, } \\
\text { alcohólicos anónimos }\end{array}$ \\
\hline 17 & Salud & $\begin{array}{l}\text { Hospital, consultorio médico, clínica dental, farmacias, } \\
\text { veterinarias }\end{array}$ \\
\hline 18 & Educación & Guarderías, escuela, instituto, academias \\
\hline 19 & Servicios & Salón de belleza, lavanderías \\
\hline 20 & Talleres & Mecánicos, ebanistería, enderezado y pintura, automotriz \\
\hline 21 & Ferreterías & Centros de pintura, cerrajería \\
\hline 22 & Oficinas Extraterritoriales & Embajadas \\
\hline 23 & Oficinas del Gobierno & Oficina gubernamental administrativa \\
\hline 24 & Vehículos & Repuestos, venta de nuevos y usados, autolavado, llantas \\
\hline
\end{tabular}

Fuente: Elaboración propia. 
Cuadro 3. Distribución de los sitios de muestreo para el sector comercial totalizado para los cantones seleccionados

\begin{tabular}{|l|c|}
\hline \multicolumn{1}{|c|}{ Actividad Comercial } & Número de muestras \\
\hline Otros servicios: financieros & 8 \\
\hline Otros servicios: religiosos & 17 \\
\hline Otros servicios: transporte & 72 \\
\hline Industria o producción & 78 \\
\hline Almacén o depósito & 2 \\
\hline Asistencia social & 6 \\
\hline Información y comunicación & 52 \\
\hline Abarrotes & 138 \\
\hline Comercio general & 90 \\
\hline Comidas y bebidas & 38 \\
\hline Entretenimiento & 22 \\
\hline Hospedaje & 14 \\
\hline Asociaciones & 48 \\
\hline Salud & 34 \\
\hline Educación & 180 \\
\hline Servicios & 38 \\
\hline Talleres & 8 \\
\hline Ferreterías & 7 \\
\hline Oficina extraterritorial & 25 \\
\hline Oficinas del gobierno & 33 \\
\hline Vehículos & $\mathbf{9 2 5}$ \\
\hline Total & 27 \\
\hline
\end{tabular}

Fuente: Elaboración propia.

Previo a la obtención de las muestras de residuos sólidos, se encuestaron, tanto los domicilios como los comercios, con el fin de recabar información referente al número de generadores en la fuente, días de la semana que se produce residuos, manejo interno de los mismos en la fuente, lugar y tipo de recipientes de almacenamiento, entre otros. Adicionalmente, se explicó la metodología del proyecto, se colocó una calcomanía de identificación en cada sitio y se realizó la entrega de las bolsas de polietileno, según la cantidad necesaria por vivienda o comercio, con su respectivo código. 
Los muestreos se programaron durante siete días consecutivos, entre los meses de agosto 2014 - diciembre 2015. A partir del segundo al sétimo día del muestreo se recogieron las bolsas que contenían los residuos generados el día anterior, entregando una nueva para la disposición durante las siguientes 24 horas. El octavo día, únicamente, se recolectaron las bolsas del día anterior. Una vez obtenidas las muestras, se realizó el pesaje de cada bolsa individual, corroborando el estrato socioeconómico al cual corresponda o la clasificación comercial asignada. Posteriormente, se llevó a cabo el cuarteo del material colectado, para esto las bolsas fueron vaciadas en un área plana horizontal de $4 \times 4 \mathrm{~m}$ de cemento provista de techo. Los residuos fueron mezclados con ayuda de una pala y rastrillos con el fin de homogeneizarlos. Una vez finalizada esta etapa, se dividieron en cuatro partes lo más iguales posible, estas fueron identificadas con A, B, C y D; y seguido se eliminaron las opuestas; es decir, A y C o B y D. Con las partes seleccionadas se realizó nuevamente el cuarteo y así sucesivamente hasta que se logró alcanzar un mínimo de $50 \mathrm{~kg}$.

\section{b. Tasa de generación}

El valor de la generación per-cápita de residuos sólidos (kg/hab-día) para domicilios se obtuvo dividiendo el peso de la bolsa entre el número de habitantes de la casa y la cantidad de trabajadores registrados para el comercio.

\section{c. Clasificación}

De los $50 \mathrm{~kg}$ obtenidos durante el cuarteo, se ejecutó la selección de los subproductos ,según lo establece el cuadro 4. Los residuos se pesaron por categoría. 
Cuadro 4. Clasificación de residuos sólidos ordinarios utilizada para la determinación de la composición

\begin{tabular}{|l|l|}
\hline \multicolumn{1}{|c|}{ Categoría } & \multicolumn{1}{c|}{ Subcategoría } \\
\hline Papel y cartón & Papel blanco y de color, periódico, cartón, cartoncillo, otros papeles \\
\hline Plástico & PET, LDPE, HDPE, otros plásticos \\
\hline Metales & Aluminio, ferrosol, no ferrosol \\
\hline Textiles & \\
\hline Polilaminados & \\
\hline Peligrosos & \\
\hline Electrónico y eléctrico & \\
\hline Vidrio & \\
\hline Biodegradables & Cáscaras, residuos de jardín, otros biodegradables \\
\hline Otros & \\
\hline
\end{tabular}

Fuente: Elaboración propia.

\section{d. Peso volumétrico}

Según lo establece la norma mexicana NOM-AA-19-1985, se tomó una muestra de las partes rechazadas del cuarteo y se llenó un estañón hasta el máximo. Este recipiente se pesó en una báscula primero vacío y, posteriormente, junto con los residuos contenidos. Para determinar el peso volumétrico se restó la masa del contenedor al valor obtenido del mismo junto con los residuos.

Al llenarse el recipiente se golpeó contra el suelo tres veces dejándolo caer de aproximadamente, $10 \mathrm{~cm}$ de altura, fueron agregados nuevamente los residuos al recipiente hasta que se llenó al máximo.

El peso volumétrico del residuo sólido se calculó mediante la siguiente fórmula:

$$
P_{v}=\frac{m}{V}
$$

En donde:

$P_{v}=$ Peso volumétrico del residuo sólido, en $\mathrm{kg} / \mathrm{m}^{3}$

$m=$ Masa de los residuos sólidos (masa bruta menos estañón), en kg

$V=$ Volumen del recipiente, en $\mathrm{m}^{3}$. 


\section{e. Análisis de laboratorio}

Se tomó una muestra de $10 \mathrm{~kg}$ a partir de las dos primeras partes eliminadas del cuarteo, según lo establece la NMX-AA-15-1985. Al llegar las muestras al laboratorio, los residuos se vaciaron en un área limpia y se procedió a cortarlos con tijeras hasta que tuvieran un tamaño máximo de $5 \mathrm{~cm}$. Los residuos se homogenizaron con una pala pequeña y por medio del cuarteo se extrajo una muestra representativa de $1 \mathrm{~kg}$, la cual se trituró hasta obtener un tamaño semejante al de arena gruesa.

De los residuos triturados se extrajo la cantidad necesaria para la determinación de humedad, los restantes se depositaron en frascos color ámbar de cuello esmerilado de 2 litros de capacidad y se almacenaron a $4^{\circ} \mathrm{C}$ para realizar los demás análisis.

Para la determinación de la humedad, se colocó una cápsula en la estufa por un periodo de dos horas. Luego, la cápsula se desecó igualmente por dos horas o hasta alcanzar peso constante. La muestra se vertió en la cápsula hasta la mitad del volumen de la misma y posteriormente, se pesó e introdujo en la estufa por dos horas. Una vez finalizada esta etapa se dejó enfriar y se pesó.

La fórmula utilizada para estimar la humedad fue la siguiente

$$
H=\frac{G-G_{1}}{G} \times 100
$$

Donde:

$H=$ Humedad en $\%$

$G=$ Masa de la muestra humedad en $\mathrm{g}$

$G_{1}=$ Masa de la muestra seca en $\mathrm{g}$

La determinación del $\mathrm{pH}$ se realizó colocando $10 \mathrm{~g}$ de muestra en un beaker de $250 \mathrm{ml}$, a los cuales se les añadió agua destilada. Luego se mezclaron y se dejaron reposar. Posteriormente, se sumergieron los electrodos y se realizó la medición. 


\section{Resultados}

En el cuadro 5, se pueden observar los valores promedio de la tasa de generación de residuos sólidos para el sector domiciliar, obtenidos según la muestra determinada para cada estrato social y cantón.

Cuadro 5. Resultados de tasas de generación por estrato socioeconómico para los cantones incluidos en el estudio

\begin{tabular}{|l|c|c|c|c|c|c|}
\hline \multicolumn{7}{|c|}{ Tasa de generación (kg/hab-día) } \\
\hline Cantón & $\begin{array}{c}\text { Estrato } \\
\text { Alto }\end{array}$ & $\begin{array}{c}\text { Estrato } \\
\text { Medio }\end{array}$ & $\begin{array}{c}\text { Estrato } \\
\text { Bajo }\end{array}$ & Rural & $\begin{array}{c}\text { Promedio } \\
\text { ponderado }\end{array}$ & $\begin{array}{c}\text { Desviación } \\
\text { estándar }\end{array}$ \\
\hline San José & 0,75 & 0,68 & 0,55 & & 0,66 & 0,43 \\
\hline Barva & 0,59 & 0,57 & 0,51 & 0,47 & 0,56 & 0,37 \\
\hline Belén & 0,81 & 0,52 & 0,36 & & 0,58 & 0,50 \\
\hline Alajuela & 0,59 & 0,51 & 0,47 & 0,65 & 0,52 & 0,46 \\
\hline \multicolumn{7}{|c|}{ Promedio ponderado Regional } \\
\hline
\end{tabular}

Fuente: Elaboración propia.

Tal como se evidencia en el cuadro 5, para todos los cantones, el estrato bajo presenta una tasa de generación promedio de residuos por habitante menor a las restantes categorías, esta diferencia puede ser explicada por el hecho de que los hábitos de consumo están determinados en gran medida por el poder adquisitivo del sector. El promedio ponderado regional de tasa de generación es de $0,59 \mathrm{~kg} / \mathrm{hab}$-día y resultó ser menor al valor reportado en el Diagnóstico de Áreas Prioritarias realizado por el PRESOL en el 2007, en donde se estimó una tasa de generación de 1 kg/hab-día.

En la figura 2, se muestra la variabilidad en los datos de la tasa de generación de residuos, en el sector domiciliar para los cantones evaluados, dependiendo del día de la semana. De lunes a sábado, la tasa de generación se distribuye entre $0,57-0,60 \mathrm{~kg} / \mathrm{hab}$-día, mostrando cierta constancia en el comportamiento. Sin embargo, en lo que corresponde al domingo, se aprecia una reducción de la tasa de generación, llegando al valor mínimo de $0,55 \mathrm{~kg} / \mathrm{hab}$-día; esto puede ser el resultado de un comportamiento generalizado de las familias por destinar el domingo como día de descanso, salir de sus casas y consumir alimentos fuera de ellas. 
Figura 2. Variación diaria de la tasa de generación de residuos en el sector domiciliar para el cantón de San José

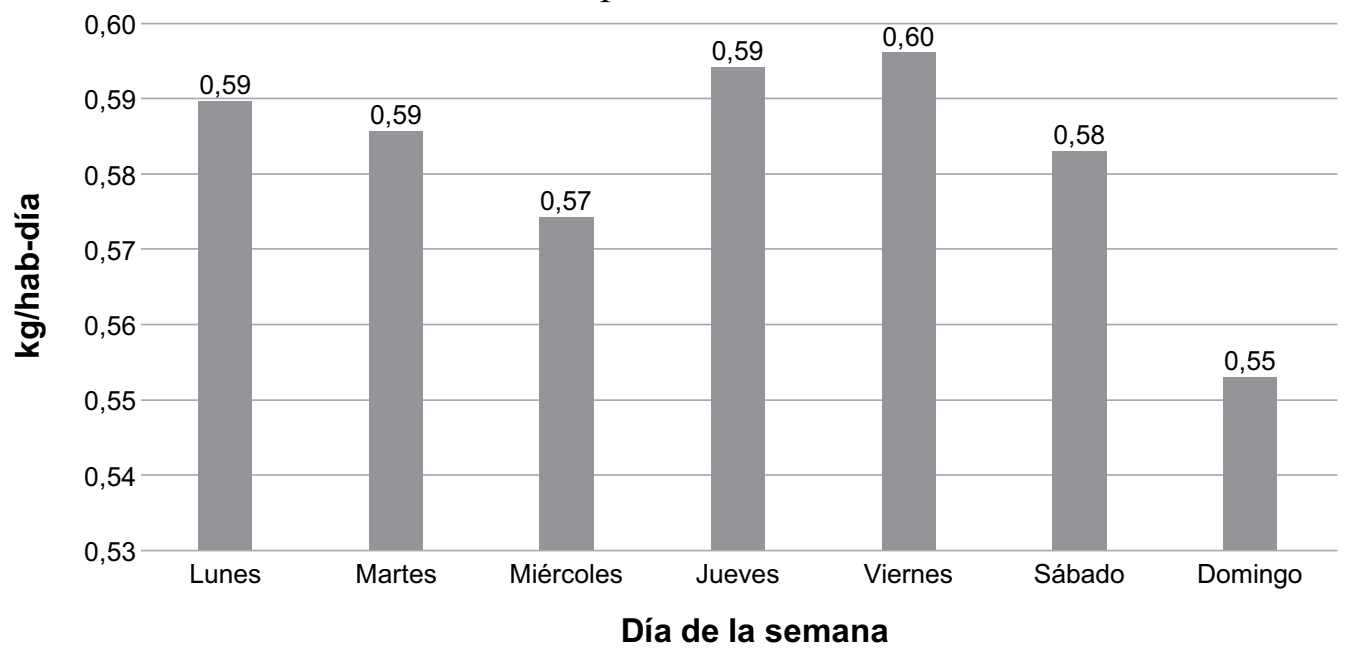

Fuente: Elaboración propia.

Adicionalmente, el valor de la tasa de generación de residuos mostró variaciones dependiendo del distrito analizado para todos los cantones estudiados. Por ejemplo, para el cantón de San José, se consideró como el sector central, a los distritos de Carmen, Hospital, Catedral y Merced; el norte corresponde a la Uruca; el sur está conformado por Hatillo y San Sebastián; el este por Zapote y San Francisco; y por último, el oeste por Mata Redonda y Pavas. Con respecto a la clasificación según estratos sociales y distribución geográfica anteriormente mencionada para la clase alta, el sector oeste es el que presenta una mayor tasa de generación, con un promedio de $0,85 \mathrm{~kg} / \mathrm{hab}$-día; por el contrario, la clase alta del sector sur es la que presenta un menor tasa, con $0,62 \mathrm{~kg} / \mathrm{hab}$-día. Para la clase media, el sector oeste se sigue manteniendo como el mayor generador, con 0,73 $\mathrm{kg} / \mathrm{hab}$-día, seguido por los sectores este, centro, sur y norte, con $0,62 \mathrm{~kg} /$ hab-día. En la generación por parte de la clase baja, se dan cambios importantes; los sectores del centro y este son los mayores generadores con 0,58 $\mathrm{kg} / \mathrm{hab}$-día. Los datos anteriores se muestran detalladamente en la figura 3. 
Figura 3. Variación de las tasas de generación domiciliares por sectores para el cantón de San José

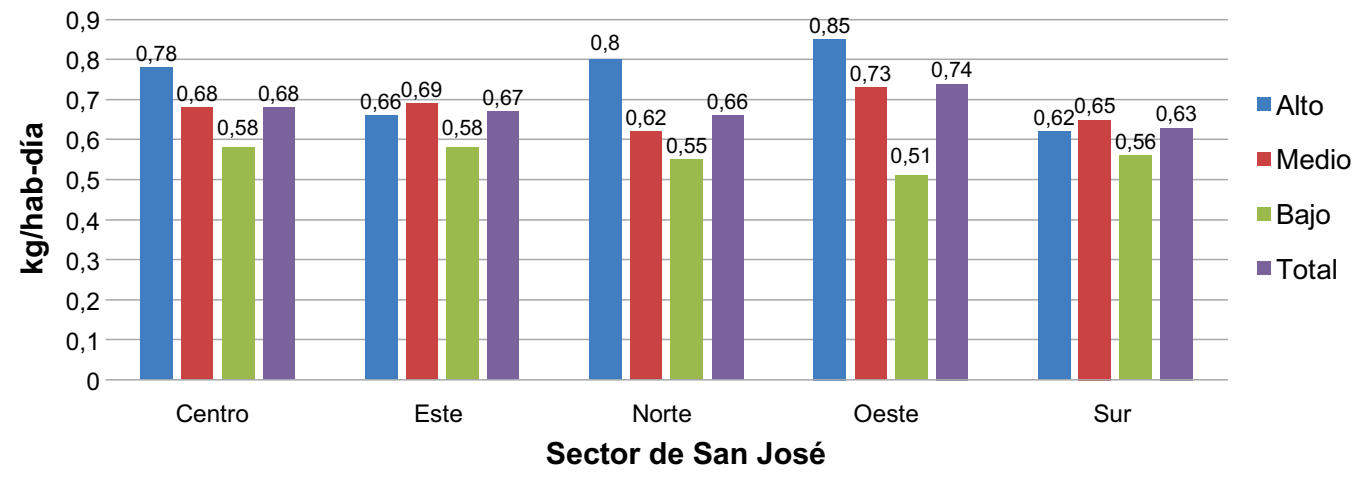

Fuente: Elaboración propia.

Con respecto al sector comercial, el cuadro 6 muestra la tasa de generación obtenida para las distintas categorías de comercio en cada uno de los cantones muestreados. Tal como se puede observar, los establecimientos de comidas y abarrotes son los entes generadores con mayores tasas.

La figura 4 muestra la generación de residuos del sector comercial, según el día de la semana. Se puede evidenciar un comportamiento constante entre semana, con una disminución el día viernes y un aumento significativo el sábado.

Al igual que en el caso domiciliar, la tasa de generación en el sector comercial presentó variaciones importantes a nivel de distritos según su ubicación espacial. Siguiendo con el ejemplo del cantón de San José, dada la alta densidad de los comercios ubicados en los distritos centrales (figura 5), es este espacio donde se obtuvo el valor promedio más alto, con 0,90 $\mathrm{kg}$ /empleado-día. Seguidamente, se encuentran los comercios del sector sur de San José, con una tasa de generación de $0,80 \mathrm{~kg} / \mathrm{empleado-día.} \mathrm{Por}$ último, en orden decreciente se encuentran los sectores norte, oeste y este, en donde este último cuenta con un valor de $0,62 \mathrm{~kg} / \mathrm{hab}$-día. 
Cuadro 6. Resultados de tasas de generación por actividad económica para el sector comercial de los cantones incluidos en el estudio

\begin{tabular}{|l|l|c|c|c|c|c|}
\hline \multirow{2}{*}{ Código } & \multirow{2}{*}{ Clasificación } & \multicolumn{5}{|c|}{ Tasa de generación (kg/empleado -día) } \\
\cline { 3 - 7 } & & San José & Belén & Barva & Alajuela & Promedio \\
\hline $\mathbf{0 4}$ & Otros servicios: Financieros & 0,172 & 0,231 & - & 0,275 & 0,226 \\
\hline $\mathbf{0 5}$ & Otros servicios: Religiosos & 1,045 & 1,218 & - & 2,398 & 1,554 \\
\hline $\mathbf{0 6}$ & Otros servicios: Transporte & 0,633 & - & 0,489 & 0,428 & 0,517 \\
\hline $\mathbf{0 7}$ & Industria o producción & 0,815 & - & - & 1,854 & 1,334 \\
\hline $\mathbf{0 8}$ & Almacén o depósito & 0,568 & - & 0,713 & - & 0,640 \\
\hline $\mathbf{0 9}$ & Asistencia social & 0,160 & - & - & 1,129 & 0,644 \\
\hline $\mathbf{1 0}$ & Información y Comunicación & 0,330 & - & - & - & 0,330 \\
\hline $\mathbf{1 1}$ & Abarrotes & 1,440 & 1,703 & - & - & 1,572 \\
\hline $\mathbf{1 2}$ & Comercio general & 0,770 & 1,109 & 1,097 & 1,604 & 1,145 \\
\hline $\mathbf{1 3}$ & Comidas y bebidas & 2,115 & 1,955 & 2,130 & 2,022 & 2,056 \\
\hline $\mathbf{1 4}$ & Entretenimiento & 0,721 & 1,274 & 0,995 & 1,692 & 1,170 \\
\hline $\mathbf{1 5}$ & Hospedaje & 0,848 & 0,773 & 0,880 & 0,486 & 0,747 \\
\hline $\mathbf{1 6}$ & Asociaciones & 0,230 & - & - & 0,915 & 0,572 \\
\hline $\mathbf{1 7}$ & Salud & 0,422 & 0,830 & - & - & 0,626 \\
\hline $\mathbf{1 8}$ & Educación & 0,732 & 0,893 & 0,766 & 1,208 & 0,899 \\
\hline $\mathbf{1 9}$ & Servicios & 0,680 & 1,428 & & 1,514 & 1,207 \\
\hline $\mathbf{2 0}$ & Talleres & 0,959 & - & 1,055 & 1,434 & 1,149 \\
\hline $\mathbf{2 1}$ & Ferreterías & 0,480 & 0,577 & 0,958 & 1,454 & 0,867 \\
\hline $\mathbf{2 2}$ & Oficinas Extraterritoriales & 0,293 & - & 0,511 & 0,687 & 0,497 \\
\hline $\mathbf{2 3}$ & Oficinas del Gobierno & 0,213 & 0,180 & - & - & 0,196 \\
\hline $\mathbf{2 4}$ & Vehículos & 0,383 & 0,610 & - & - & 0,496 \\
\hline
\end{tabular}

Fuente: Elaboración propia. 
Figura 4. Variación del promedio diario de generación de residuos en el sector comercial para los cantones estudiados

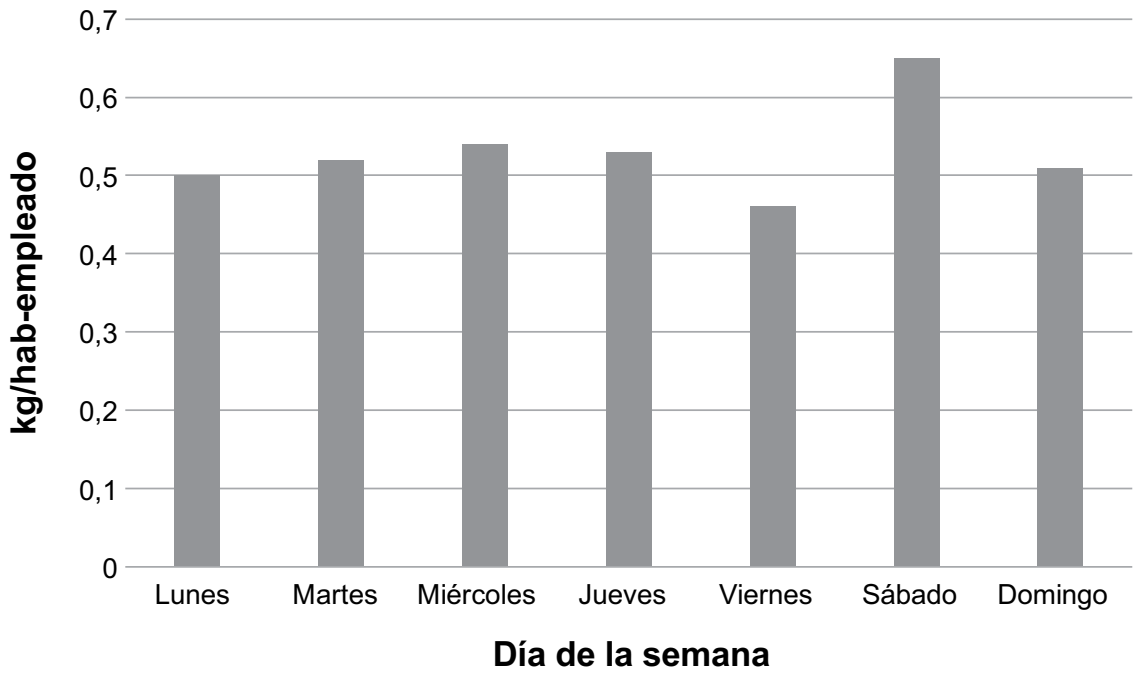

Fuente: Elaboración propia.

Figura 5. Variación espacial de la tasa de generación de residuos del sector comercial del Cantón de San José

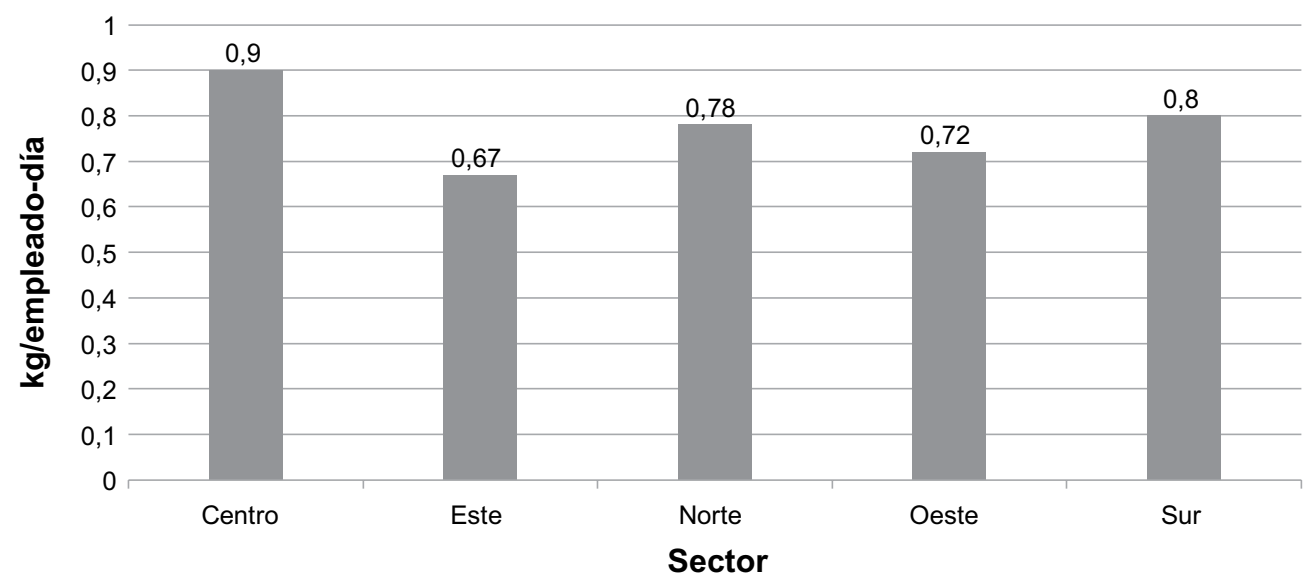

Fuente: Elaboración propia. 


\section{Caracterización de residuos sólidos}

El cuadro 7 muestra las distribuciones con respecto a la composición de los residuos sólidos provenientes del sector domiciliar y comercial. En el caso de las viviendas, los residuos que tienen mayor presencia son los biodegradables, seguidos por los denominados otros componentes. Los porcentajes de papel, cartón y plástico representan casi un $10 \%$ del total generado. Esto pone en evidencia que la mayoría de los residuos generados en los hogares pueden someterse a algún tipo de aprovechamiento antes de ser dispuestos en los rellenos sanitarios, como lo son por ejemplo, los centros de recuperación de residuos valorizables e incluso, la generación de compost derivado de los componentes orgánicos desechados. El panorama cambia cuando se analizan los datos correspondientes a los comercios muestreados. Para este caso los residuos como el plástico, el papel y el cartón tienen más importancia que en el sector domiciliar. Los empaques y envolturas utilizadas en los comercios para el manejo de los productos y servicios ofrecidos, marcan esta diferencia. Este sector tiene un mayor potencial para el aprovechamiento de los residuos sólidos en los centros de recuperación de valorizables.

En la figura 6, se puede apreciar que no hay una diferencia significativa en la caracterización de los residuos domiciliares por estrato socioeconómico para el área de estudio. Sin embargo, a nivel espacial (distritos) si se presentan algunas variaciones importantes que se deben tomar en cuenta a la hora de desarrollar propuestas de revalorización de residuos, tal es el caso del cantón de San José (figura 7). 
Jorge Herrera-Murillo, José Félix Rojas-Marín, Deivis Anchía-Leitón. Tasas de generación y caracterización de residuos sólidos ordinarios en cuatro municipios del Área Metropolitana Costa Rica

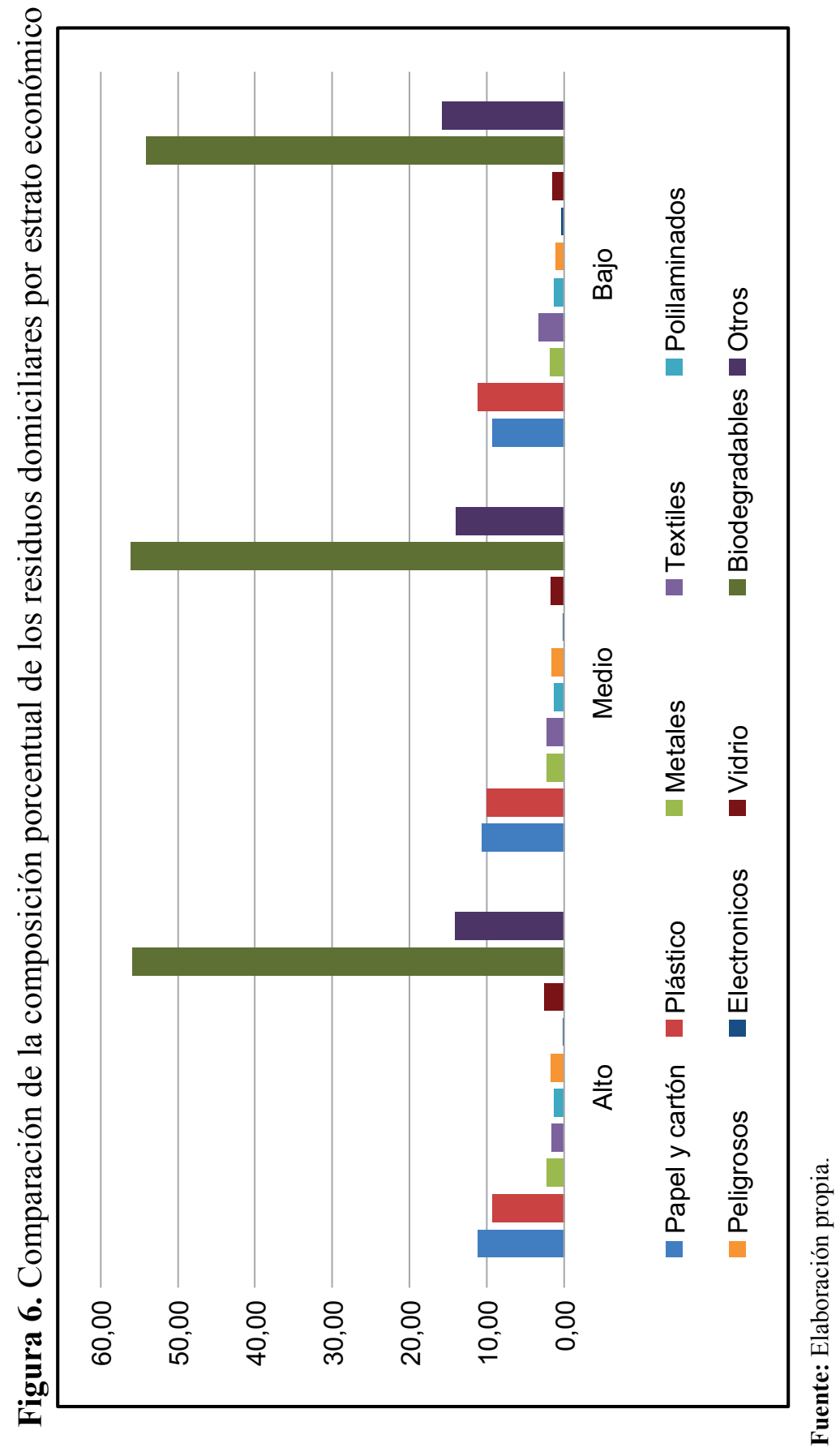


Jorge Herrera-Murillo, José Félix Rojas-Marín, Deivis Anchía-Leitón. Generation rates and characterization of ordinary solid waste in four municipalities of the Metropolitan Area Costa Rica

Cuadro 7. Resultados de la caracterización de residuos (\%) para los sectores domiciliar y comercial de los cantones incluidos en el estudio

\begin{tabular}{|c|c|c|c|c|c|c|c|c|c|}
\hline \multirow{2}{*}{\multicolumn{2}{|c|}{$\begin{array}{c}\text { Caracterización en } \\
\text { porcentaje }\end{array}$}} & \multicolumn{2}{|c|}{ San José } & \multicolumn{2}{|c|}{ Belén } & \multicolumn{2}{|c|}{ Barva } & \multicolumn{2}{|c|}{ Alajuela } \\
\hline & & Domicilio & Comercio & Domicilio & Comercio & Domicilio & Comercio & Domicilio & Comercio \\
\hline \multirow{5}{*}{ 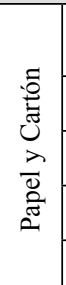 } & \begin{tabular}{l|}
$\begin{array}{l}\text { Papel blanco y } \\
\text { de color }\end{array}$ \\
\end{tabular} & 1,3 & 3,0 & 0,2 & 0,9 & 0,6 & 0,6 & 0,2 & 1,6 \\
\hline & Periódico & 2,5 & 1,1 & 0,9 & 1,0 & 0,8 & 0,3 & 0,3 & 0,8 \\
\hline & Cartón & 1,5 & 11,4 & 1,3 & 10,6 & 1,1 & 8,5 & 0,6 & 14,7 \\
\hline & Cartoncillo & 1,8 & 3,3 & 1,2 & 3,8 & 1,5 & 4,6 & 1,2 & 5,5 \\
\hline & Otros papeles & 3,3 & 8,5 & 2,7 & 5,2 & 3,7 & 3,6 & 5,8 & 11,7 \\
\hline \multirow{4}{*}{ 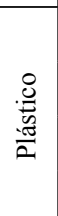 } & PET & 1,5 & 2,9 & 1,2 & 1,9 & 0,9 & 1,1 & 1,6 & 1,7 \\
\hline & HDPE & 1,1 & 1,4 & 2,5 & 3,0 & 4,1 & 5,5 & 5,1 & 4,2 \\
\hline & LDPE & 4,1 & 3,3 & 0,8 & 2,4 & 0,3 & 1,5 & 0,5 & 1,8 \\
\hline & Otros plásticos & 3,5 & 7,9 & 4,6 & 5,2 & 4,0 & 4,8 & 3,0 & 4,4 \\
\hline \multirow{3}{*}{$\frac{0}{\frac{0}{\pi}}$} & Aluminio & 0,6 & 0,4 & 0,4 & 0,5 & 0,8 & 0,4 & 0,4 & 0,4 \\
\hline & Ferroso & 1,2 & 1,7 & 0,8 & 0,7 & 1,1 & 2,8 & 1,8 & 0,8 \\
\hline & No ferroso & 0,2 & 0,0 & 0,4 & 0,0 & 0,1 & 0,0 & 0,0 & 0,0 \\
\hline \multicolumn{2}{|c|}{ Textiles } & 2,4 & 2,9 & 2,5 & 2,6 & 2,1 & 3,1 & 3,8 & 1,7 \\
\hline \multicolumn{2}{|c|}{ Polilaminados } & 1,3 & 1,4 & 1,0 & 1,2 & 1,1 & 0,7 & 1,1 & 1,0 \\
\hline \multicolumn{2}{|c|}{ Peligrosos } & 1,5 & 1,7 & 1,5 & 1,4 & 0,3 & 1,1 & 0,4 & 0,1 \\
\hline \multicolumn{2}{|c|}{ Electrónicos } & 0,2 & 1,3 & 0,04 & 0,4 & 1,5 & 0,3 & 0,2 & 0,6 \\
\hline \multicolumn{2}{|c|}{ Vidrio } & 1,9 & 1,3 & 1,9 & 0,9 & 1,8 & 0,4 & 1,1 & 0,7 \\
\hline \multirow{3}{*}{ 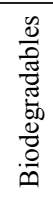 } & Cáscaras & 25,7 & 12,0 & 31,1 & 20,6 & 33,6 & 32,7 & 34,2 & 20,1 \\
\hline & Jardín & 2,6 & 3,3 & 8,9 & 2,7 & 8,5 & 0,0 & 2,4 & 1,8 \\
\hline & $\begin{array}{l}\text { Otros } \\
\text { biodegradables }\end{array}$ & 27,0 & 11,9 & 16,6 & 16,5 & 14,7 & 18,7 & 14,9 & 12,4 \\
\hline \multicolumn{2}{|c|}{ Otros componentes } & 14,7 & 19,4 & 19,6 & 18,5 & 17,5 & 9,2 & 21,5 & 14,0 \\
\hline \multicolumn{2}{|c|}{ Total } & 100 & 100 & 100 & 100 & 100 & 100 & 100 & 100 \\
\hline
\end{tabular}

Fuente: Elaboración propia. 
Figura 7. Variación espacial de la caracterización de residuos para el sector domiciliar del cantón de San José

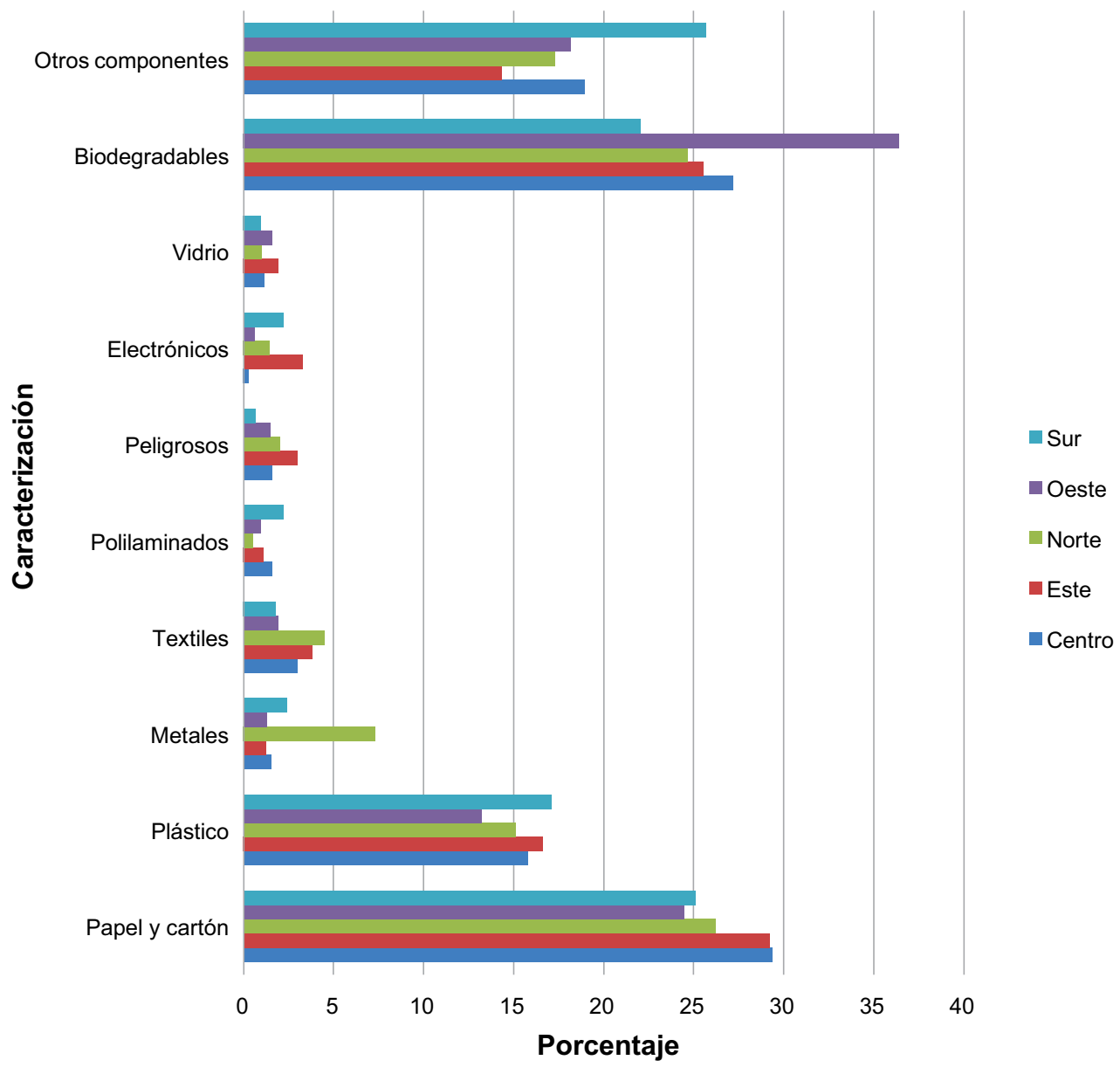

Fuente: Elaboración propia.

\section{Peso volumétrico}

Con respecto al peso volumétrico (cuadro 8), los residuos presentaron valores promedio de 155 y $73 \mathrm{~kg} / \mathrm{m}^{3}$ para el sector domiciliar y comercial, respectivamente. Esta diferencia, se puede fundamentar en los resultados de la caracterización de los residuos sólidos, ya que los provenientes de los domicilios presentan un mayor porcentaje de materia orgánica, y este componente aporta un importante peso a la medición. De la misma forma, los 
del sector comercial son más voluminosos que los domiciliares, por las características de las actividades que se desarrollan. Se puede afirmar que la cantidad de residuos orgánicos contenidos en una muestra aumentan el valor del peso volumétrico, y se espera que aquellas muestras con altos valores de peso volumétrico tengan mayor contenido de residuos orgánicos.

No se presentaron diferencias significativas en los valores reportados para los diferentes cantones.

Cuadro 8. Resultados de la determinación del peso volumétrico $\left(\mathrm{kg} / \mathrm{m}^{3}\right)$ de los residuos generados en el área de estudio

\begin{tabular}{|l|c|c|c|c|}
\hline \multicolumn{1}{|c|}{ Sector } & San José & Belén & Barva & Alajuela \\
\hline Domiciliar & $163 \pm 14$ & $141 \pm 37$ & $158 \pm 35$ & $159 \pm 40$ \\
\hline Comercial & $84 \pm 21$ & $55 \pm 36$ & $61 \pm 37$ & $93 \pm 29$ \\
\hline
\end{tabular}

Fuente: Elaboración propia.

\section{EI valor reportado con \pm representa la desviación estándar del dato}

\section{pH}

Los valores de $\mathrm{pH}$ resultantes (Cuadro 9) de los análisis químicos reflejan que ambos sectores analizados producen residuos moderadamente ácidos, siendo los provenientes de los comercios los que presentan mayor acidez en comparación con los del sector domiciliar.

Cuadro 9. Resultados de la determinación de $\mathrm{pH}$ de los residuos sólidos comerciales y domiciliarios generados en el área de estudio

\begin{tabular}{|l|c|c|c|c|}
\hline \multicolumn{1}{|c|}{ Sector } & San José & Belén & Barva & Alajuela \\
\hline Domiciliar & $5,45 \pm 0,62$ & $5,72 \pm 0,57$ & $5,13 \pm 0,41$ & $5,31 \pm 0,43$ \\
\hline Comercial & $5,19 \pm 0,87$ & $5,14 \pm 0,38$ & $4,87 \pm 0,27$ & $5,10 \pm 0,35$ \\
\hline
\end{tabular}

Fuente: Elaboración propia.

El valor reportado con \pm representa la desviación estándar del dato

Es importante considerar que los residuos que presentan un $\mathrm{pH}$ alejado a 7, limitan el crecimiento de los microorganismos, lo que eventualmente puede afectar la descomposición de los mismos y su utilización en formas de tratamiento como el compostaje. Las bacterias, por ejemplo, 
son microorganismos que crecen en ambientes donde se presente un $\mathrm{pH}$ entre 6 y 7,5, a diferencia de los hongos que si poseen un mayor margen de tolerancia para su desarrollo, entre 5 y 8.

\section{Humedad}

El contenido de humedad en los residuos sólidos se refiere al porcentaje de agua que estos contienen. Según Colomer y Gallardo (2013), los factores que influyen en el porcentaje de agua de los residuos son, entre otros: el contenido de materia orgánica, la procedencia, la forma en que se presentan y la climatología de la región. Con respecto a los datos obtenidos en las muestras (cuadro 10) analizadas, los residuos comerciales presentan menor porcentaje de humedad que los domiciliares, esto debido a que la humedad está relacionada con el porcentaje de residuos orgánicos que estos contengan y; por ende, una mayor probabilidad de contaminar los adyacentes y aportarles humedad. Tomando en cuenta aspectos teóricos expuestos por Colomer y Gallardo (2013), la humedad promedio oscila entre un 35-65\%, y puede llegar a extremos máximos como por ejemplo residuos de mercados (60-80\%). Cuando los residuos se quieren utilizar para procesos de compostaje, el rango ideal se encuentra entre un $45 \mathrm{y}$ $55 \%$. Los datos de humedad obtenidos resultaron ser muy homogéneos entre los cantones incluidos en el estudio.

Cuadro 10. Resultados de la determinación de humedad de los residuos sólidos comerciales y domiciliarios generados en el área de estudio

\begin{tabular}{|l|c|c|c|c|}
\hline \multicolumn{1}{|c|}{ Sector } & San José & Belén & Barva & Alajuela \\
\hline Domiciliar & $66 \pm 10$ & $63 \pm 7$ & $66 \pm 10$ & $67 \pm 6$ \\
\hline Comercial & $58 \pm 11$ & $60 \pm 8$ & $63 \pm 8$ & $54 \pm 7$ \\
\hline
\end{tabular}

Fuente: Elaboración propia.

\section{El valor reportado con \pm representa la desviación estándar del dato}

El dato del porcentaje de humedad de los residuos sólidos, es de vital importancia para poder calcular la cantidad de calor que se puede recuperar a partir de ellos, ya que si el contenido en humedad es alto mucha energía será consumida al eliminar el agua. Adicionalmente, la humedad debe ser tomada en cuenta para diseñar los requerimientos necesarios para el transporte de los mismos. 


\section{Conclusiones}

En los cantones seleccionados, un determinado patrón de distribución espacial de los residuos está asociado a las motivaciones propias de una sociedad de consumo que se mueve y localiza en el espacio para satisfacer ciertas necesidades de sobrevivencia u ocio, quienes posteriormente, debido a sus actividades productivas pasaron a transformarse en los generadores de residuos, y al mismo tiempo, están marcando una demanda espacial de un sistema de gestión integral de residuos sólidos.

En cuanto a la generación y caracterización de residuos en los cuatro municipios del GAM analizados se presentan como componentes mayoritarios: orgánicos $55,9 \%$, seguidos de materiales con alto potencial para ser reciclados o utilizados como combustibles (plásticos 10,2\%, papel y cartón $10,4 \%$ ). De esta forma, si se procede al realizar la separación de la fracción orgánica de los residuos, la restante sería una mezcla con un alto contenido de componentes con capacidad a ser reciclados o utilizados en procesos de combustión. Llama la atención que en los municipios muestreados se encontraron porcentajes pequeños de residuos considerados como peligrosos, los cuales deberían ser gestionados de manera diferenciada y no forman parte de la corriente de residuos sólidos ordinarios. Al comparar los resultados obtenidos para los sectores comerciales y residenciales se puede evidenciar que en el primer caso, los materiales con potencial de reciclaje adquieren una mayor preponderancia que los residuos de naturaleza orgánica.

Tanto los gobiernos locales incluidos como los restantes dentro del GAM y el territorio nacional, deben fomentar la separación completa de la fracción orgánica con el fin de impulsar el manejo integral de los residuos sólidos en cada uno de los cantones. De esta forma, se debe lograr una reducción significativa del contenido en la humedad de los mismos, que disminuya la cantidad de lixiviados y facilite su transporte y posterior manejo. Adicionalmente, esta variación en el porcentaje de humedad aumentaría la cantidad de energía por unidad de masa que podría generarse en un proceso que involucre su combustión.

Los resultados obtenidos acerca de las propiedades físicas y químicas de los residuos sólidos, dejan en evidencia que los municipios cuentan con un gran porcentaje de producción de residuos que pueden ser sometidos a un proceso de valorización de materiales, el cual permita disminuir la cantidad de residuos que actualmente se envían hacia los rellenos

Revista Geográfica de América Central No 57 ISSN 1011-484X, julio-diciembre 2016, pp. 235-260 
sanitarios $\mathrm{u}$ otras formas de disposición final mayormente contaminantes para el ambiente. Cabe resaltar, que actualmente, las poblaciones han desarrollado cierto nivel de intolerancia hacia estos sitios, por lo que incluso la localización espacial de los mismos ocasiona implicaciones a nivel municipal y un problema en la planificación del desarrollo urbano.

Además, esos resultados son un insumo importante para facilitar el proceso de toma de decisiones y permite valorar la posibilidad de incorporar otros mecanismos para el tratamiento y valorización de materiales que se adapte a las políticas de desarrollo establecidas a nivel nacional y que actualmente se utilizan en otras latitudes.

Finalmente, los estudios de tasas de generación y caracterización de residuos sólidos representan una herramienta importante para las municipalidades, les permite diseñar sistemas de tratamiento y la optimización del recurso económico y humano. Así mismo, representan un insumo de vital importancia dentro del proceso de planificación urbana y ordenamiento territorial en los cantones costarricenses, ya que permiten calcular la vida útil de los rellenos sanitarios y anticiparse a la localización espacial de uno nuevo en el peor de los casos o prepararse para incorporar un nuevo sistema de manejo de residuos sólidos, mediante el cual se logre disminuir los impactos negativos que reciben los recursos naturales del país.

\section{Agradecimientos}

Los autores reconocen el valioso aporte realizado por las municipalidades de los cuatro cantones participantes, sin los cuales no se podría haber desarrollado el presente estudio.

\section{Referencias}

Castillo, A. (2013). Propuesta conceptual del aprovechamiento de los residuos sólidos orgánicos municipales generados en el estado de Morelos mediante la tecnología de digestión (tesis de licenciatura). Universidad Nacional Autónoma de México, México.

Colomer, F. y Gallardo, A. (2013). Tratamiento y gestión de residuos sólidos. España: Universidad Politécnica de Valencia. (pág 35-38).

Hoornweg, D. y Bhada-Tata, P. (2012). What a Waste: A Global Review of Solid Waste Management. Washington, D.C.: World Bank. 
Jaramillo, J. (2002). Guía para el diseño, construcción y operación de rellenos sanitarios manuales. Perú: Centro Panamericano de Ingeniería Sanitaria y Ciencias del Ambiente

La Gaceta. (29 de Junio de 2016). La Gaceta. Recuperado de http://www. gaceta.go.cr/pub/2010/07/13/COMP_13_07_2010.html

López-Acosta, A. (1999). Manual para muestreos de Residuos Sólidos. Memoria profesional. Escuela Superior de Ingeniería y Arquitectura, Instituto Politécnico Nacional, México

Lund, H. y Muenster, M. (2010). Comparing Waste-to-Energy technologies by applying energy system analysis. Waste Management, 30, 1251-1263.

Programa Competitividad y Medio Ambiente (CYMA). (2007). Plan de Residuos Sólidos Costa Rica (PRESOL): Diagnóstico. s/e. San José, Costa Rica.

Programa Competitividad y Medio Ambiente (CYMA). (2012). Guía metodológica para la realización de estudios de caracterización de residuos. San José, Costa Rica.

SEMARNAT. (2006). Diagnostico Básico para la Gestión Integral de Residuos. México: Secretaria de Medio Ambiente y Recursos Naturales.

Tchobanoglous, G., Theisen, H. y Vigil, S. (1994). Gestión integral de residuos sólidos. New York: McGraw-Hill.

Vértice, P. (2008). Gestión medioambiental: manipulación de residuos y productos químicos. Colombia: Publicaciones Vértice. 